\title{
A Strictly Improved Achievable Region for Multiple Descriptions Using Combinatorial Message Sharing
}

\author{
Kumar Viswanatha, Emrah Akyol and Kenneth Rose \\ ECE Department, University of California - Santa Barbara \\ \{kumar,eakyol,rose\}@ece.ucsb.edu
}

\begin{abstract}
We recently proposed a new coding scheme for the L-channel multiple descriptions (MD) problem for general sources and distortion measures involving 'Combinatorial Message Sharing' (CMS) [7| leading to a new achievable ratedistortion region. Our objective in this paper is to establish that this coding scheme strictly subsumes the most popular region for this problem due to Venkataramani, Kramer and Goyal (VKG) [3]. In particular, we show that for a binary symmetric source under Hamming distortion measure, the CMS scheme provides a strictly larger region for all $L>2$. The principle of the CMS coding scheme is to include a common message in every subset of the descriptions, unlike the VKG scheme which sends a single common message in all the descriptions. In essence, we show that allowing for a common codeword in every subset of descriptions provides better freedom in coordinating the messages which can be exploited constructively to achieve points outside the VKG region.
\end{abstract}

Index Terms-Multiple descriptions coding, Source coding, Rate distortion theory

\section{INTRODUCTION}

The Multiple Descriptions (MD) problem has been studied extensively since late 1970s, see [1], [2], [3], [4], [5], [6] and the references therein. In a $L$-descriptions MD setup, the encoder sends $L$ packets (descriptions) which are sent to the receiver over $L$ different channels. In the most general setting, it is assumed that the decoder receives a subset of the descriptions without any error and the remaining are completely lost. The decoder reconstructs the source upto a given level of distortion when a subset of the descriptions are received. The goal of the MD problem is to establish the complete rate-distortion region to trade-off the encoding rates to the achievable distortions. The general setup has remained challenging and unsolved due to the intricacies of the problem in maintaining the balance between the full reconstruction quality versus quality of individual descriptions.

Until recently, for general sources and distortion measures, the most recognized achievable rate-distortion region for the $L$-channel MD setup was due to Venkataramani, Kramer and Goyal (VKG) [3], whose encoding scheme builds on the prior work for the 2-channel case by El-Gamal and Cover (EC) [1] and Zhang and Berger (ZB) [2]. The VKG scheme involves a combinatorial number of refinement codebooks along with a single shared codebook used to control the redundancy across the descriptions. We introduced a new encoding scheme in

The work was supported by the NSF under grants CCF-0728986 and CCF $-1016861$
[7 $]^{1}$ involving 'Combinatorial Message Sharing' (CMS) which differs from the VKG scheme primarily in the number of shared codebooks. The CMS scheme allows for every subset of the descriptions to share a different common codebook, thereby leading to a combinatorial number of shared messages. At the time of submission of [7], it was not known whether the CMS scheme leads to a strictly improved rate-distortion region over the VKG scheme. In this paper, our objective is to prove by example that the new region is indeed strictly better. Specifically, we show that for a binary symmetric source under Hamming distortion measure, the CMS scheme achieves points outside the VKG region $\forall L>2$. In fact, more generally, our result holds $\forall L>2$ for any source and distortion measures for which the $\mathrm{ZB}$ scheme achives points outside the EC scheme for the corresponding 2-descriptions problem. We note in passing that, other encoding schemes have been proposed in the literature for certain special cases (specific sources and distortion measures) of the $L$-channel MD setup [6], which achieve points outside $\mathcal{R} \mathcal{D}_{V K G}$. However, none of these schemes have been proven to subsume or outperform $\mathcal{R} \mathcal{D}_{V K G}$ for general sources and distortion measures. The potential implications of our results on these coding schemes are beyond the scope of this paper. In the following Section, we formally state the $L$-channel MD setup and describe the prior results due to EC [1], ZB [2], VKG [3] and the CMS scheme [7]. In section III], we prove the strict improvement of the achievable region.

\section{Formal Definitions AND PRIOR RESUlts}

We follow the notation in [7]. A source produces $n$ iid copies, denoted by $X^{n}=\left(X^{(1)}, X^{(2)} \ldots, X^{(n)}\right)$, of a generic random variable $X$ taking values in a finite alphabet $\mathcal{X}$. We denote $\mathcal{L}=\{1, \ldots, L\}$. There are $L$ encoding functions, $f_{l}(\cdot) l \in \mathcal{L}$, which map $X^{n}$ to the descriptions $J_{l}=f_{l}\left(X^{n}\right)$, where $J_{l} \in\left\{1, \ldots B_{l}\right\}$ for some $B_{l}>0$. The rate of description $l$ is defined as $R_{l}=\log _{2}\left(B_{l}\right)$. Each of the descriptions are sent over a separate channel and are either received at the decoder error free or are completely lost. There are $2^{L}-1$ decoding functions for each possible received combination of the descriptions $\hat{X}_{\mathcal{K}}^{n}=\left(\hat{X}_{\mathcal{K}}^{(1)}, \ldots, \hat{X}_{\mathcal{K}}^{(n)}\right)=g_{\mathcal{K}}\left(J_{l}: l \in \mathcal{K}\right)$, $\forall \mathcal{K} \subseteq \mathcal{L}, \mathcal{K} \neq \phi$, where $\hat{X}_{\mathcal{K}}$ takes on values on a finite set $\hat{\mathcal{X}}_{\mathcal{K}}$, and $\phi$ denotes the null set. When a subset $\mathcal{K}$ of the descriptions are received at the decoder, the distortion is measured

${ }^{1}$ For the benefit of the reviewers, the submitted version of [7] is available at : http://www.scl.ece.ucsb.edu/Kumar/ISIT_MD_Sub.pdf 


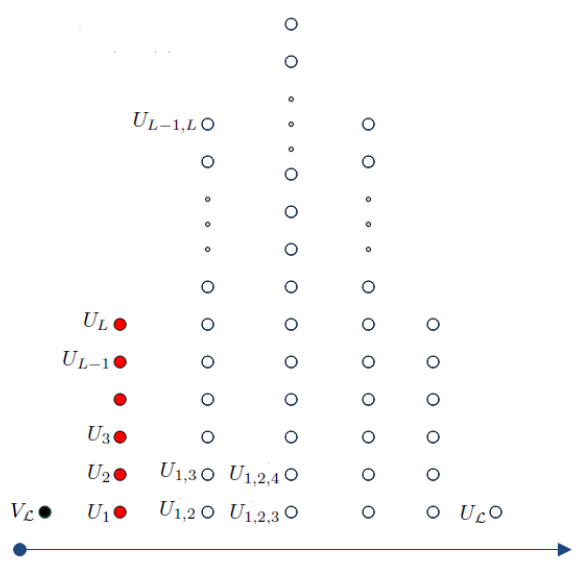

Figure 1: Codebook generation for VKG coding scheme. Black $\left(V_{\mathcal{L}}\right)$ indicates 'common random variable'. $\operatorname{Red}\left(U_{l} l \in \mathcal{L}\right)$ indicates 'base layer random variables' and White $\left(U_{\mathcal{S}}|\mathcal{S}|>1\right)$ indicates 'refinement random variables'. The arrow indicates the order of codebook generation.

as $D_{\mathcal{K}}=E\left[\frac{1}{N} \sum_{t=1}^{n} d_{\mathcal{K}}\left(X^{(t)}, \hat{X}_{\mathcal{K}}^{(t)}\right)\right]$ for some bounded distortion measures $d_{\mathcal{K}}(\cdot)$ defined as $d_{\mathcal{K}}: \mathcal{X} \times \hat{\mathcal{X}}_{\mathcal{K}} \rightarrow \mathcal{R}$. We say that a rate-distortion tuple $\left(R_{i}, D_{\mathcal{K}}: i \in \mathcal{L}, \mathcal{K} \subseteq\right.$ $\mathcal{L}, \mathcal{K} \neq \phi)$ is achievable if there exit $L$ encoding functions with rates $\left(R_{1} \ldots, R_{L}\right)$ and $2^{L}-1$ decoding functions yielding distortions $D_{\mathcal{K}}$. The closure of the set of all achievable rate-distortion tuples is defined as the ' $L$-channel multiple descriptions $R D$ region'. Note that, this region has $L+2^{L}-1$ dimensions.

In what follows, $2^{\mathcal{S}}$ denotes the set of all subsets (power set) of any set $\mathcal{S}$ and $|\mathcal{S}|$ denotes the set cardinality. Note that $\left|2^{\mathcal{S}}\right|=2^{|\mathcal{S}|}$. $\mathcal{S}^{c}$ denotes the set complement. For two sets $\mathcal{S}_{1}$ and $\mathcal{S}_{2}$, we denote the set difference by $\mathcal{S}_{1}-\mathcal{S}_{2}=\{\mathcal{K}: \mathcal{K} \in$ $\left.\mathcal{S}_{1}, \mathcal{K} \notin \mathcal{S}_{2}\right\}$. We use the shorthand $\{U\}_{\mathcal{S}}$ for $\left.\left\{U_{\mathcal{K}}: \mathcal{K} \in \mathcal{S}\right\}\right\}^{2}$

\section{A. VKG Encoding Scheme}

The achievable region of [3] is denoted here $\mathcal{R} \mathcal{D}_{V K G}$ and is described as follows. Let $\left(V_{\mathcal{L}},\{U\}_{2^{L}-\phi}\right)$ be any set of $2^{L}$ random variables distributed jointly with $X$. Then, an RD tuple is said to be achievable if there exist functions $\psi_{\mathcal{S}}(\cdot)$ such that:

$$
\begin{aligned}
\sum_{l \in \mathcal{S}} R_{l} \geq & |\mathcal{S}| I\left(X ; V_{\mathcal{L}}\right)-H\left(\{U\}_{2^{\mathcal{S}}-\phi} \mid X, V_{\mathcal{L}}\right) \\
& +\sum_{\mathcal{K} \subseteq \mathcal{S}} H\left(U_{\mathcal{K}} \mid\{U\}_{2} \mathcal{K}_{-\phi-\mathcal{K}}\right) \\
D_{\mathcal{S}} \geq & E\left[d_{\mathcal{S}}\left(X, \psi_{\mathcal{S}}\left(V_{\mathcal{L}},\{U\}_{2^{\mathcal{S}}-\phi}\right)\right)\right]
\end{aligned}
$$

$\forall \mathcal{S} \subseteq \mathcal{L}$. The closure of the achievable tuples over all such $2^{L}$ random variables gives $\mathcal{R} \mathcal{D}_{V K G}$. Here, we only present an overview of the encoding scheme. The order of codebook generation of the auxiliary random variables is shown in Figure 1 First, $2^{n R_{\mathcal{L}}^{\prime \prime}}$ codewords of $V_{\mathcal{L}}$ are generated using the marginal distribution of $V_{\mathcal{L}}$. Conditioned on each codeword of $V_{\mathcal{L}}, 2^{n R_{l}^{\prime}}$ codewords of $U_{l}$ are generated

\footnotetext{
${ }^{2}$ Note the difference between $\{U\}_{\mathcal{S}}$ and $U_{\mathcal{S}} .\{U\}_{\mathcal{S}}$ is a set of variables, whereas $U_{\mathcal{S}}$ is a single variable.
}

according to their respective conditional densities. Next, for each $j \in\left(1, \ldots, 2^{n\left(R_{\mathcal{L}}+\sum_{l \in \mathcal{K}} R_{l}^{\prime}\right)}\right)$, a single codeword is generated for $U_{\mathcal{K}}(j)$ conditioned on $\left(v_{\mathcal{L}}(j),\{u(j)\}_{2^{\mathcal{K}}-\phi-\mathcal{K}}\right)$ $\forall \mathcal{K} \subseteq \mathcal{L},|\mathcal{K}|>1$. Note that to generate the codebook for $U_{\mathcal{K}}$, we first need the codebooks for all $\{U\}_{2} \mathcal{K}_{-\phi-\mathcal{K}}$ and $V_{\mathcal{L}}$.

On observing a typical sequence $x^{n}$, the encoder tries to find a jointly typical codeword tuple one from each codebook. Codeword index of $U_{l}$ (at rate $R_{l}^{\prime}$ ) is sent in description $l$. Along with the 'private' messages, each description also carries a 'shared message' at rate $R_{\mathcal{L}}^{\prime \prime}$, which is the codeword index of $V_{\mathcal{L}}$. Hence the rate of each description is $R_{l}=R_{l}^{\prime}+$ $R_{\mathcal{L}}^{\prime \prime}$. VKG showed that, to ensure finding a set of jointly typical codewords with the observed sequence, the rates must satisfy (1). It then follows from standard arguments (see for example "typical average lemma" [8]) that, if the random variables also satisfy (2), then the distortion constraints are met. Note that, $V_{\mathcal{L}}$ is the only shared random variable. $U_{l}: l \in \mathcal{L}$ form the base layer random variables and all $U_{\mathcal{K}}:|\mathcal{K}| \geq 2$ form the refinement layers. Observe that the codebook generation follows the order: shared layer $\rightarrow$ base layer $\rightarrow$ refinement layer.

The VKG scheme for the 2-descriptions scenario involves 4 auxiliary random variables $V_{12}, U_{1}, U_{2}$ and $U_{12}$. The VKG region was originally derived as an extension of the EC [1] and ZB [2] coding schemes, which were designed for the 2descriptions scenario. The first of the two regions was by El-Gamal and Cover and their rate region (denoted here by $\left.\mathcal{R} \mathcal{D}_{E C}\right)$ is obtained by setting $V_{12}=\Phi$ in $\mathcal{R} \mathcal{D}_{V K G}$, where $\Phi$ is a constant. Zhang and Berger (their region is denoted here by $\mathcal{R} \mathcal{D}_{Z B}$ ) later showed that, including the shared random variable can give strict improvement over $\mathcal{R} \mathcal{D}_{E C}$. Their result, while perhaps counter-intuitive at first, clarifies the fact that, a shared message among the descriptions helps to better coordinate the messages, thereby providing a strictly improved $\mathrm{RD}$ region, even though it introduces redundancy. We will describe their result in detail in Section [III as our example builds upon theirs. However, it is known that $\mathcal{R} \mathcal{D}_{E C}$ is complete for some special cases of the setup (see for example [4], [8]).

\section{B. CMS Encoding Scheme}

In this section, we briefly describe our CMS encoding scheme in [7]. The VKG encoding scheme employs one common codeword $\left(V_{\mathcal{L}}\right)$ that is sent in all the $L$ descriptions. However, when dealing with $L>2$ descriptions, restricting to a single shared message could be suboptimal. The CMS scheme therefore allows for 'combinatorial message sharing', i.e a common codeword is sent in each (non-empty) subset of the descriptions. Before describing the codebook generation and stating the theorem, we define the following subsets of $2^{\mathcal{L}}$ :

$$
\begin{aligned}
\mathcal{I}_{W} & =\left\{\mathcal{S}: \mathcal{S} \in 2^{\mathcal{L}},|\mathcal{S}|=W\right\} \\
\mathcal{I}_{W+} & =\left\{\mathcal{S}: \mathcal{S} \in 2^{\mathcal{L}},|\mathcal{S}|>W\right\}
\end{aligned}
$$




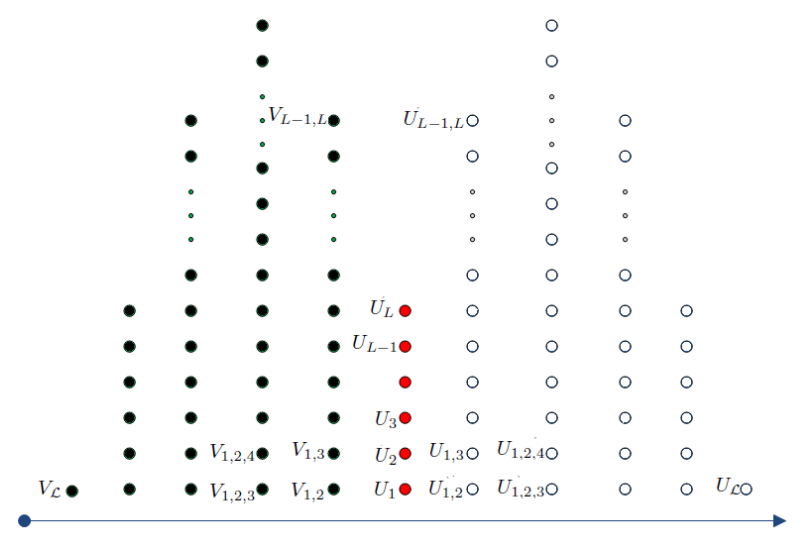

Figure 2: Codebook generation for the CMS coding scheme.

Let $\mathcal{B}$ be any non-empty subset of $\mathcal{L}$ with $|\mathcal{B}| \leq W$. We define the following subsets of $\mathcal{I}_{W}$ and $\mathcal{I}_{W+}$ :

$$
\begin{aligned}
\mathcal{I}_{W}(\mathcal{B}) & =\left\{\mathcal{S}: \mathcal{S} \in \mathcal{I}_{W}, \mathcal{B} \subseteq \mathcal{S}\right\} \\
\mathcal{I}_{W+}(\mathcal{B}) & =\left\{\mathcal{S}: \mathcal{S} \in \mathcal{I}_{W+}, \mathcal{B} \subseteq \mathcal{S}\right\}
\end{aligned}
$$

We also define 3 .

$\mathcal{J}(\mathcal{K})=\bigcup_{l \in \mathcal{K}} \mathcal{I}_{1+}(l)=\{\mathcal{S}: \mathcal{S} \subseteq \mathcal{L},|\mathcal{S}|>1,|\mathcal{K} \cap \mathcal{S}|>0\}$

The shared random variables are denoted by ' $V$ '. The base and the refinement layer random variables are denoted by ' $U$ '. The codebook generation is done in an order as shown in Figure 2. First, the codebook for $V_{\mathcal{L}}$ is generated. Then, the codebooks for $V_{\mathcal{S}},|S|=W$ are generated in the order $W=L-1, L-2 \ldots 2.2^{n R_{\mathcal{Q}}^{\prime \prime}}$ codewords of $V_{\mathcal{Q}}$ are independently generated conditioned on each codeword tuple of $\{V\}_{\mathcal{I}_{W+}(\mathcal{Q})}$. This is followed by the generation of the base layer codebooks, i.e. $U_{l}, l \in \mathcal{L}$. Conditioned on each codeword tuple of $\{V\}_{I_{1+}(l)}, 2^{n R_{l}^{\prime}}$ codewords of $U_{l}$ are generated independently. Then the codebooks for the refinement layers are formed by generating a single codeword for $U_{\mathcal{S}},|\mathcal{S}|>1$ conditioned on every codeword tuple of $\left(\{V\}_{\mathcal{J}(\mathcal{S})},\{U\}_{2} \mathcal{S}_{-\mathcal{S}}\right)$. Observe that the base and the refinement layers in the CMS scheme are similar to that in the VKG scheme, except that they are now generated conditioned on a subset of the shared codewords.

The encoder employs joint typicality encoding, i.e., on observing a typical sequence $x^{n}$, it tries to find a jointly typical codeword tuple, one from each codebook. As with the VKG scheme, the codeword index of $U_{l}$ (at rate $R_{l}^{\prime}$ ) is sent in description $l$. However, now the codeword index of $V_{\mathcal{S}}$ (at rate $R_{\mathcal{S}}^{\prime \prime}$ ) is sent in all the descriptions $l \in \mathcal{S}$. Therefore the rate of description $l$ is:

$$
R_{l}=R_{l}^{\prime}+\sum_{\mathcal{K} \in \mathcal{J}(l)} R_{\mathcal{K}}^{\prime \prime}
$$

We next state the main result in [7] which describes a new region for the $L-$ Channel MD setup achievable by the CMS scheme. Let $\left(\{V\}_{\mathcal{J}(\mathcal{L})},\{U\}_{2^{\mathcal{L}}-\phi}\right)$ be any set of $2^{L+1}-L-2$

\footnotetext{
${ }^{3}$ We use the notation $\mathcal{A} \subseteq \mathcal{B}$ to mean $\mathcal{A}$ is subsumed in $\mathcal{B}$ and $\mathcal{A} \subset \mathcal{B}$ to mean strictly subsumed.
}

random variables jointly distributed with $X$. We define the quantities $\alpha_{W}(\mathcal{Q})$ and $\beta(\mathcal{S})$ as follows:

$$
\begin{aligned}
\alpha_{W}(\mathcal{Q})= & \sum_{\mathcal{S} \in \mathcal{Q}} H\left(V_{\mathcal{S}} \mid\{V\}_{\mathcal{I}_{W+}(\mathcal{S})}\right) \\
& -H\left(\{V\}_{\mathcal{Q}} \mid\{V\}_{\mathcal{I}_{W+}}, X\right) \quad \forall \mathcal{Q} \subseteq \mathcal{I}_{W} \\
\beta(\mathcal{S})= & \sum_{\mathcal{K} \subseteq \mathcal{S}} H\left(U_{\mathcal{K}} \mid\{U\}_{2^{\mathcal{K}}-\phi-\{\mathcal{K}\}},\{V\}_{\mathcal{J}(\mathcal{K})}\right) \\
& -H\left(\{U\}_{2^{\mathcal{S}}-\phi} \mid\{V\}_{\mathcal{I}_{1+}}, X\right) \quad \forall \mathcal{S} \subseteq \mathcal{L}
\end{aligned}
$$

We follow the convention $\alpha_{W}(\phi)=\beta(\phi)=0$. Let $R_{\mathcal{K}}^{\prime \prime} \forall \mathcal{K} \in$ $\mathcal{J}(\mathcal{L})$ and $R_{l}^{\prime} \forall l \in \mathcal{L}$ be any set of rate tuples satisfying:

$$
\begin{aligned}
\sum_{\mathcal{K} \in \mathcal{Q}} R_{\mathcal{K}}^{\prime \prime} & >\alpha_{W}(\mathcal{Q}) \forall \mathcal{Q} \subseteq \mathcal{I}_{W}, W \in \mathcal{L} \\
\sum_{l \in \mathcal{S}} R_{l}^{\prime} & >\beta(\mathcal{S}) \forall \mathcal{S} \subseteq \mathcal{L}
\end{aligned}
$$

then, the RD region for the $L$-channel MD problem contains the rates and distortions for which there exist functions $\psi_{\mathcal{S}}(\cdot)$, such that

$$
\begin{aligned}
R_{l} & \geq R_{l}^{\prime}+\sum_{\mathcal{K} \in \mathcal{J}(l)} R_{\mathcal{K}}^{\prime \prime} \\
D_{\mathcal{S}} & \geq E\left[d_{\mathcal{S}}\left(X, \psi_{\mathcal{S}}\left(\{V\}_{\mathcal{J}(\mathcal{S})},\{U\}_{2^{\mathcal{S}}-\phi}\right)\right)\right]
\end{aligned}
$$

The closure of the achievable tuples over all such $2^{L+1}-L-2$ random variables is denoted by $\mathcal{R} \mathcal{D}_{C M S}$. Observe that both the VKG and the CMS schemes are same as the ZB scheme for 2 descriptions scenario.

\section{PROOF OF STRICT IMPROVEMENT}

Note that, the total number of auxiliary random variables in the CMS scheme is almost twice that in the VKG scheme (which already is exponential in $L$ ). At the time of submission of [7], it was yet unclear if this increase pays off with an improved achievable region. The following theorem, being the main contribution of this paper, establishes that there exists scenarios for which $\mathcal{R} \mathcal{D}_{C M S}$ is strictly larger than $\mathcal{R} \mathcal{D}_{V K G}$.

Theorem 1. (i) The rate-distortion region achievable by the CMS scheme is always at least as large as the region achievable by the VKG region, i.e.:

$$
\mathcal{R D}_{V K G} \subseteq \mathcal{R D}_{C M S}
$$

(ii) There exists scenarios for which the CMS scheme leads to a region strictly larger than that achievable by the VKG scheme, i.e.:

$$
\mathcal{R D}_{V K G} \neq \mathcal{R D}_{C M S} \Rightarrow \mathcal{R D}_{V K G} \subset \mathcal{R} \mathcal{D}_{C M S}
$$

Specifically, for a binary symmetric source under Hamming distortion measure, the CMS scheme achieves a strictly larger rate-distortion region compared to the VKG scheme $\forall L>2$.

Proof: Part (i) of the theorem is rather simple to prove and is a straight forward corollary of the main theorem in [7]. It follows directly by setting $V_{\mathcal{S}}=\Phi \forall \mathcal{S}$ such that $|\mathcal{S}|<L$ in $\mathcal{R} \mathcal{D}_{C M S}$. We then have $\alpha_{W}(\mathcal{Q})=0 \forall \mathcal{Q}, W<L$. Substituting in $\sqrt{9}$, we get $R_{(\mathcal{S})} \geq \beta(\mathcal{S})+|\mathcal{S}| \alpha_{L}(\mathcal{L})$ which is same as $\sqrt{2}$. 
We prove (ii) by considering the binary symmetric source example for which the CMS scheme achieves points which cannot be achieved by the VKG scheme. Note that, once we prove that the CMS scheme achieves a strictly larger region for some $L=l>2$, then it must be true for all $L \geq l$. Hence to prove (ii), it is sufficient for us to show that it is true for $L=3$. However, we first include an example for $L=4$ for building intuition and understanding of the type of scenarios where the CMS scheme provides strict improvement. Then we will prove the result for $L=3$. We also note that obviously scenarios exit for which $\mathcal{R} \mathcal{D}_{C M S}=\mathcal{R} \mathcal{D}_{V K G}$ (for example when $\mathcal{R} \mathcal{D}_{V K G}$ is complete [3]). Finding the set of all such scenarios is an interesting problem in itself and is beyond the scope of this paper. To describe our example, we require certain results pertaining to binary multiple descriptions [2] and successive refinement of binary sources [9], [10]. In what follows, we state these results.

The Zhang-Berger example : Zhang and Berger proved that, for the binary symmetric 2-descriptions MD problem under Hamming distortion measure, sending a common codeword in both the descriptions provides a strict improvement over the EC scheme. We briefly describe their result. Note that the rate-distortion region has 5 dimensions denoted by $\left(R_{1}, R_{2}, D_{1}, D_{2}, D_{12}\right)$. Denote the rate-distortion region achievable by the $\mathrm{EC}$ scheme by $\mathcal{R D}_{E C}(\operatorname{bern}(1 / 2))$ and the corresponding region achievable by the ZB scheme (i.e. achieved by adding a common codeword among the two descriptions) by $\mathcal{R} \mathcal{D}_{Z B}(\operatorname{bern}(1 / 2)$. Obviously $\mathcal{R D}_{E C}(\operatorname{bern}(1 / 2)) \subseteq \mathcal{R D}_{Z B}(\operatorname{bern}(1 / 2))$, as we can always choose not to send any common codeword in the ZB scheme. Denote by $\bar{R}_{E C}(D)$ the following cross section of $\mathcal{R} \mathcal{D}_{E C}(\operatorname{bern}(1 / 2))$ :

$$
\begin{gathered}
\bar{R}_{E C}(D)=\inf \left\{R_{1}+R_{2}: \quad D_{1}+D_{2} \leq 2 D\right. \\
\left.\left(R_{1}, R_{2}, D_{1}, D_{2}, 0\right) \in \mathcal{R D}_{E C}(\operatorname{bern}(1 / 2))\right\}
\end{gathered}
$$

Similarly, denote by $\bar{R}_{Z B}(D)$, the corresponding cross section of $\mathcal{R D}_{Z B}(\operatorname{bern}(1 / 2))$. To show that $\mathcal{R} \mathcal{D}_{E C}(\operatorname{bern}(1 / 2)) \subset$ $\mathcal{R D}_{Z B}$ (bern $\left.(1 / 2)\right)$, they considered a particular joint probability mass function (PMF) $P^{*}\left(X, V_{12}, U_{1}, U_{2}, U_{12}\right)$. Let us denote the achievable region associated with this PMF by $\mathcal{R D}_{P^{*}}(\operatorname{bern}(1 / 2))$ and the corresponding cross-section 13 by $\bar{R}_{P^{*}}(D)$. They showed that $\exists D^{*}>0$ such that $R_{E C}\left(D^{*}\right)>\bar{R}_{P^{*}}\left(D^{*}\right) \geq \bar{R}_{Z B}\left(D^{*}\right)$. We refer the reader to [2] for a detailed derivation and the values of $P^{*}$ and $D^{*}$.

Successive refinement : The problem of successive refinement was first proposed by Equitz and Cover in [9] and has since then been studied extensively by information theorists [9], [10]. The problem is motivated by scalable coding, where the encoder generates two layers of information called the base layer and the enhancement layer. The base layer provides a coarse reconstruction of the source, while the enhancement layer is used to 'refine' the reconstruction beyond the base layer. The objective is to encode the two layers such that the distortion at both the base and the enhancement layers are optimal. This setup is shown schematically in Figure 3a Observe that, the 2-layer successive refinement region is indeed a special case (the cross-section $\left(R_{1} \cdot R_{2}, D_{1}, \infty, D_{12}\right)$ ) of the 2-descriptions MD setup where the distortion constraint on one of the individual descriptions is removed. The complete rate region for successive refinement was derived in [10] where it was shown that the EC coding scheme achieves the complete rate region.

An interesting followup question is that of 'successive refinability' of sources. Assume $d_{1}=d_{2}=d$, then a source is said to be successively refinable under $d$ if $\forall D_{1} \geq D_{2}$, the rate point $\left(R_{1}, R_{2}\right)=\left(\mathcal{R D}_{s}\left(D_{1}\right), \mathcal{R D}_{s}\left(D_{2}\right)-\mathcal{R D}_{s}\left(D_{1}\right)\right)$ is achievable, where $\mathcal{R} \mathcal{D}_{s}(D)$ denotes Shannon's rate distortion function. This condition implies that there is no loss in describing the source in two successive parts. An important point to note is that, for a successively refinable source, when the encoder operates at $\left(R_{1}, R_{2}\right)=\left(\mathcal{R D}_{s}\left(D_{2}\right), \mathcal{R D}_{s}\left(D_{2}\right)-\right.$ $\left.\mathcal{R} \mathcal{D}_{s}\left(D_{1}\right)\right)$, there is absolutely no redundancy between the two layers of information, i.e., the two layers cannot carry a common codeword. We finally note that a binary symmetric source is successively refinable under Hamming measure [9].

Proof of (ii) : $\mathrm{L}=4$ : Consider a 4-descriptions MD problem for a binary symmetric source $\left(\operatorname{bern}\left(\frac{1}{2}\right)\right)$ under Hamming distortion measure. The rate-distortion region consists of 19 dimensions. We denote the region achievable by the VKG scheme by $\mathcal{R D}_{V K G}^{4}$ and that achievable using the CMS scheme by $\mathcal{R D}_{C M S}^{4}$. We now consider a particular cross-section of these regions where we apply constraints only on $D_{1}, D_{2}, D_{12}, D_{3}$ and $D_{34}$. We remove the constraints on all other distortions, i.e. we set $D_{4}, D_{13}, D_{14}, D_{23}, D_{24} D_{123}, D_{124}, D_{134}, D_{234}$ and $D_{1234}$ to $\infty$. Equivalently, we can think of a 4 descriptions MD problem with a particular channel failure pattern, wherein only one of the following sets of descriptions can reach the decoder reliably: $(1,2,3,\{1,2\},\{3,4\})$ as shown in Figure $3 b$ We denote the set of all achievable points for this setup using the VKG and the CMS schemes by $\widetilde{\mathcal{D D}}_{V K G}^{4}$ and $\widehat{\mathcal{R D}}_{C M S}^{4}$ respectively. Note that, this equivalent model is used simply for analysis purposes, while we are actually interested in a cross section of the general binary symmetric 4-descriptions region.

Observe that, with respect to the first 2 descriptions, we have a simple 2-descriptions problem and with respect to the last 2 descriptions, we have a successive refinement problem. Extending the arguments of Zhang and Berger, we define the following infimum of $\widetilde{\mathcal{R D}}_{V K G}^{4}$ :

$$
\begin{array}{r}
\tilde{R}_{V K G}^{4}(D)=\inf \left\{R_{1}+R_{2}: D_{1}+D_{2} \leq 2 D,\right. \\
\left(R_{1}, R_{2}, R_{3}, R_{4}, D_{1}, D_{2}, 0, D_{3}, D_{34}\right) \in \widetilde{\mathcal{R D}}_{V K G}^{4}, \\
\left.R_{3}=\mathcal{R D}_{s}\left(D_{3}\right), R_{3}+R_{4}=\mathcal{R D}_{s}\left(D_{34}\right)\right\}
\end{array}
$$

Denote the corresponding infimum of $\widetilde{\mathcal{R D}}_{C M S}^{4}$ by $\tilde{R}_{C M S}^{4}(D)$. Recall that the VKG scheme forces all the descriptions to have a single common codeword. Constraints $R_{3}=\mathcal{R D}_{s}\left(D_{3}\right)$ and $R_{3}+R_{4}=\mathcal{R D}_{s}\left(D_{34}\right)$ ensure that descriptions 3 and 4 carry completely complementary information, i.e. they cannot 


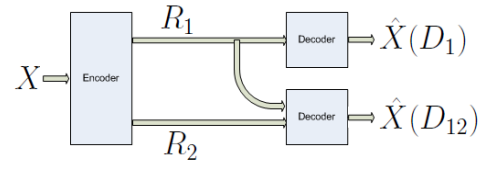

(a)

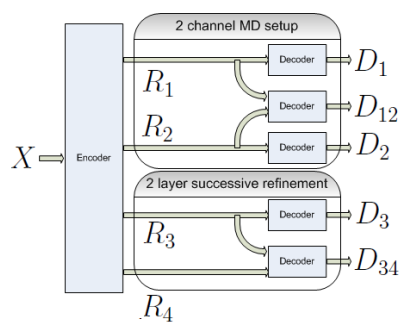

(b)

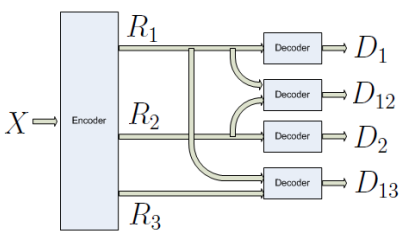

(c)

Figure 3: (a)Successive refinement setup (b) Equivalent model for the considered cross-section of the 4-descriptions setup (c) Equivalent model for the considered cross-section of the 3-descriptions setup

carry a common codeword 44 As VKG coding scheme forces the same common codeword among all the 4 descriptions, it follows that:

$$
\tilde{R}_{V K G}^{4}\left(D^{*}\right)=\bar{R}_{E C}\left(D^{*}\right)>\bar{R}_{Z B}\left(D^{*}\right)
$$

On the other hand, the CMS scheme allows for distinct common codewords to be sent in each subset of the descriptions. Hence, we can send a common codeword only among the two descriptions 1 and 2 while still maintaining $R_{3}=\mathcal{R} \mathcal{D}_{s}\left(D_{3}\right)$ and $R_{3}+R_{4}=\mathcal{R} \mathcal{D}_{s}\left(D_{34}\right)$. This is achieved by setting all the common random variables to $\Phi$ except $V_{12}$, which has joint PMF $P^{*}$ with $\left(X, U_{1}, U_{2}, U_{12}\right)$. This allows us to achieve:

$$
\tilde{R}_{C M S}^{4}\left(D^{*}\right)=\bar{R}_{Z B}\left(D^{*}\right)
$$

This implies that $\tilde{R}_{V K G}^{4}\left(D^{*}\right)>\tilde{R}_{C M S}^{4}\left(D^{*}\right)$ and hence $\mathcal{R D}_{V K G}^{4} \subset \mathcal{R D}_{C M S}^{4}$. This example clearly illustrates the freedom the CMS scheme exhibits in controlling the redundancy across the messages.

$\mathbf{L}=\mathbf{3}$ : In similar lines to the 4-descriptions case, we next consider a 3-descriptions MD problem for a binary symmetric source under Hamming distortion measure. Let the achievable regions be denote by $\mathcal{R D}_{V K G}^{3}$ and $\mathcal{R D}_{C M S}^{3}$ respectively. We consider the cross-sections of the achievable regions where we apply constraints only on $D_{1}, D_{2}, D_{12}$ and $D_{13}$ as shown in Figure $3 \mathrm{c}$. We denote these cross-sections by $\widetilde{\mathcal{R D}}_{V K G}^{3}$ and $\widetilde{\mathcal{R D}}_{C M S}^{3}$ respectively. Now consider any point $\left(R_{1}, R_{2}, R_{3}, D_{1}, D_{2}, D_{12}, D_{13}\right) \in \mathcal{R D}_{V K G}^{3}$ such that $\left(R_{1}, R_{2}, D_{1}, D_{2}, D_{12}\right) \in \mathcal{R} \mathcal{D}_{Z B}-\mathcal{R} \mathcal{D}_{E C}$ and $D_{13}<D_{1}$, where for two sets $\mathcal{A}$ and $\mathcal{B}, \mathcal{A}-\mathcal{B}=\{l: l \in \mathcal{A}, l \notin \mathcal{B}\}$. From the results of Zhang and Berger, if $\left(R_{1}, R_{2}, D_{1}, D_{2}, D_{12}\right) \in$ $\mathcal{R} \mathcal{D}_{Z B}-\mathcal{R D}_{E C}$, descriptions 1 and 2 must carry a common codeword. Let the rate of the common codeword be $R_{c}>0$. VKG scheme forces this codeword to be sent as part of $R_{3}$ as well. As this common codeword is received as part of both

\footnotetext{
${ }^{4}$ Note that, the constraint $R_{3}=\mathcal{R D}_{s}\left(D_{3}\right)$ is in fact redundant. Just the constraint $R_{3}+R_{4}=\mathcal{R D}_{s}\left(D_{34}\right)$ is sufficient to establish that descriptions 3 and 4 cannot carry a common codeword. However, as a binary source is successively refinable, we can always achieve $D_{3}=\mathcal{R D}_{s}^{-1}\left(R_{3}\right)$ and hence the constraint $R_{3}=\mathcal{R D}_{s}\left(D_{3}\right)$ gets applied implicitly once we apply $R_{3}+$ $R_{4}=\mathcal{R} \mathcal{D}_{s}\left(D_{34}\right)$. This implies that, the gains due to the CMS scheme are not only restricted to successively refinable sources. In fact, the CMS scheme can achieve points outside the VKG region for any source and distortion measure for which the ZB scheme achieves points outside the EC scheme for the corresponding 2-descriptions setup.
}

descriptions 1 and 3 , it is redundant in $R_{3}$ to achieve $D_{13}$. This implies that $\left(R_{1}, R_{2}, R_{3}-R_{c}, D_{1}, D_{2}, D_{12}, D_{13}\right) \in \mathcal{R D}_{C M S}^{3}$. As there exit points in the boundary of $\mathcal{R D}_{V K G}^{3}$ which satisfy $\left(R_{1}, R_{2}, D_{1}, D_{2}, D_{12}\right) \in \mathcal{R} \mathcal{D}_{Z B}-\mathcal{R D}_{E C}$, the CMS scheme achieves points outside the VKG scheme. Hence, we have shown that for a binary symmetric source under Hamming distortion measure, the CMS scheme achieves a strictly larger rate-distortion region than the VKG scheme for all $L>2$.

\section{CONCLusion}

We recently proposed a new encoding scheme for the general $L$-channel multiple descriptions problem involving 'combinatorial message sharing' (CMS) which leads to a new achievable region subsuming the most well known region for this problem by Venkataramani, Kramer and Goyal (VKG) [3] for general sources and distortion measures. In this paper, we showed that there exists scenarios (particularly for a binary symmetric source under Hamming distortion measure) for which, the new region is strictly larger than that achievable by the VKG scheme. As part of future work, we will investigate under what scenarios the CMS scheme achieves the complete $\mathrm{RD}$ region.

\section{REFERENCES}

[1] A. El Gamal and T. M. Cover, "Achievable rates for multiple descriptions," IEEE Trans. Inf. Theory, vol. IT-28, pp. 851-857, Nov. 1982.

[2] Z. Zhang and T. Berger, "New results in binary multiple descriptions," IEEE Trans. Inf. Theory, vol. IT-33, pp. 502-521, July 1987.

[3] R. Venkataramani, G. Kramer, and V. K. Goyal, "Multiple description coding with many channels," IEEE Trans. Inf. Theory, vol. 49, no. 9 , pp. 2106-2114, Sep. 2003.

[4] L. Ozarow, "On a source-coding problem with two channels and three receivers," Bell Syst. Tech. J., vol. 59, no. 10, pp. 1909-1921, Dec. 1980

[5] J. Wang, J. Chen, L. Zhao, P. Cuff, and H. Permuter, "A random variable substitution lemma with applications to multiple description coding," preprint. [Online]. Available: http://arxiv.org/abs/0909.3135

[6] R. Puri, S. S. Pradhan, and K. Ramchandran, "n-channel symmetric multiple descriptions-part II: an achievable rate-distortion region," IEEE Trans. Inf. Theory, vol. 51, pp. 1377-1392, Apr. 2005.

[7] K. Viswanatha, E. Akyol and K. Rose, "Combinatorial message sharing for a refined multiple descriptions achievable region," to appear at the proceedings of IEEE international symposium on information theory (ISIT) 2011. Submitted version available at : http://www.scl.ece.ucsb.edu/Kumar/ISIT_MD_Sub.pdf

[8] A. El Gamal and Y. H. Kim, "Lecture notes on network information theory", http://arxiv.org/abs/1001.3404. 2010.

[9] W. H. R. Equitz and T. M. Cover,"Successive refinement of information," IEEE Trans. Inf. Theory, vol. 37, no. 2, pp. 269-275, 1991. 
[10] B. Rimoldi, "Successive refinement of information: Characterization of the achievable rates," IEEE Trans. Inf. Theory, vol. 40, no. 1, pp. 253-259, Jan. 1994. 